\title{
Hard-wall entropic effect accelerates detachment of adsorbed polymer chains
}

\author{
Cheng-Tai Lee and Eugene M. Terentjev ${ }^{1}$ \\ Cavendish Laboratory, University of Cambridge, J.J. Thomson Avenue, Cambridge, CB3 OHE, \\ U.K.
}

(Dated: 20 August 2019)

Many previous studies of unbinding kinetics have focused on a two-state model, with fully-bonded and free states, which may not extend to more complicated biopolymer dynamics involving other reactions. Here we address the kinetic rate of this process at the segment level, as it is influenced by a growing dangling end of the chain. We use the mean first-passage time approach, and treat the polymer as a chain attached to a wall through a succession of spring potentials, with two distinct regions of bonded and free segments. The interaction between the wall and free-moving chain end adds an entropic repulsion to this process. We estimate the average monomer detachment rate $K$ as a function of the free dangling length $L$. For flexible polymer, we find an acceleration factor in the average detachment rate depending on $L$ and the details of the spring bond; when $L$ is long, this factor is a simple ratio of its breaking distance to the natural bond length. For semi-flexible filament, we examine the regime where $L$ is shorter than persistence length $L_{p}$, as the limit opposite to the flexible chain. An enhancing factor also appears, speeding up the filament unbinding when free length grows; for a long rigid rod, this factor becomes 2, independently of the bond details. We also examine the total unbinding time of an irreversible detaching process by integrating $(1 / K)$ over polymer length, and discover that its power-law scaling with chain length is smaller than one, over the commonly-seen range of polymer size.

\section{INTRODUCTION}

Detachment of adsorbed polymers from surface is a fundamental kinetic process. 1 , tant problem due to its frequent involvement in biological systems such as single biopolymers attached to cell membrane $e^{489}$. This polymer-surface system is also relevant in unzipping of two multiply bonded polymer chains or filaments such as microtubule filaments, known as one of the main components of the cellular network controlling their length through an unbinding and dissociation process 10 . Therein, one of the two laterally-bonded filaments are usually treated as a hard wall, respecting the fact that two filaments cannot penetrate each other. Due to an increasing impact of single-molecule techniques, such as atomic force microscopy and optical tweezers, experiment is able to investigate thermodynamics and kinetics of single biopolymers 13 , accordingly attracting theoretical interest to the underlying physics of sequential unbinding.

Although many of the previous studies have examined kinetics of unbinding the whole chain attached to a hard surface and unzipping of two bonded filaments $7|11| 13 \mid 17+19$, they mostly applied the two-state transition kinetic theory, considering a fully-bonded and a fully-unzipped states with a transition barrier to cross. The task became to describe the transition state: solving a Schrödinger-like equation of the end-end separation distance $e^{1314}$ or the filament-shape equation with the bonded length being the free energy minimization parameter 18 . The kinetic rate constant of unzipping is then determined by the Arrhenius activation law, proportional to the Boltzmann factor of barrier-crossing. Little effort was put into understanding the unbinding kinetics of individual monomers or chain segments during the detachment process.

This two-state simplification is somewhat unrealistic in biological phenomena, which are usually quite dynamic and accompanied by various side reactions before the entire chain fully unbinds from its substrate. Although Paturej and coworkers 7 derived an evolution equation for the unbound length by using a blob-trumpet picture of the polymer shape, it is still not applicable to incorporate the effect of accompanying reactions, as surely the inclusion of other reactions will change their evolution equation, and the new equation may be hard to find. In fact, a trend of applying the master kinetic equation method has been growing in the biopolymer area 20 . Therein, the evolution of the entire system is governed by many step-by-step reactions, described through a set of kinetic equations. This has the benefit to allow other possible intervening reactions contribute to this dynamic process. In this sense, unbinding of the entire chain will be treated as a monomer-by-monomer process, with each polymer state characterized by the length of the free unbound segment. It is therefore indispensable to find the average detachment rate constant at this monomer/segmentlevel during unbinding process of all bonded monomers, instead of using the full-filament view.

Here we address the unbinding problem at the segment level, specifically focusing on finding the monomer detachment rate. We consider a polymer chain laterally bonded to a hard surface. The unbinding process occurs from one end, and proceeds continuously by one segment at a time, without jumping (the segment will not breakoff, if the previous neighbor has not yet done so). Hence, there are only two distinct regions along the polymer, bonded and detached (the latter of the length $L$ ), see Fig. 11(A) and Fig. 3(A) below for illustration.

Under this scenario, the detached chain segments freely explore the space above the wall, subject to thermal mo- 
tion and the grafting constraint. This contributes additional entropic effect to the unbinding kinetics. Many previous studies have reported such an entropic effect due to spatial confinement, in dynamics of flexible polymers 24 and glassy colloids ${ }^{25}$, in desorption kinetics of polymeric surfactants (diblock copolymers) which forms a monolaymer with many brushes pointing out normal to the surface $^{1}$, and in stiff semi-flexible chain systems 26 . 28 . Nevertheless, this effect has not been explored in unzipping kinetics. Here, our goal is to study this entropic effect on the step-by-step unbinding kinetic rate constant in two distinct cases: for a flexible chain and for a semiflexible filament.

We will not consider a more complicated situation where some of the segments along the free unbound chain may have re-attached back onto the surface, forming loops on the dangling chain. Our aim in this paper is purely to calculate the rate constant to detach the lastbonded monomer with a free dangling chain without reattachment The purpose is to establish some of the unbinding rate constants required for applying the master equation method to describe dynamics of fully detaching an adsorbed polymer at the surface.

The paper is organized as follows: in Sec. IIA, we review the previous studies of entropic repulsion between the wall and the grafted polymer, and obtain the effective potential along the unbinding coordinate, essentially making it a one-dimensional problem. In Sec. IIB, we introduce the mean first-passage time (MFTP) method ${ }^{29130}$ in order to find out the average detachment rate of the last bonded monomer in this context. This general method is widely applied in polymer reaction theories 31132 ; the unbinding rate we obtain has a geometric pre-factor, $\operatorname{erf}\left(c / 2 R_{g}\right) / \operatorname{erf}\left(a / 2 R_{g}\right)$, increasing the escape rate of a harmonically-bonded particle, where $a$ is the natural bond length, $R_{g}$ is the radius of gyration of the unbound dangling chain segment, and $c$ is the bond-breaking length. For a very long unbound chain, this pre-factor reduces to $(c / a)$.

In Sec. III A, we consider a discrete semi-flexible filament, with bending penalty described by the KratkyPorod mode $\sqrt{33}$. In the case where the unbound chain length $L$ is shorter than the persistence length $L_{p}$, we treat the unbound chain as a rigid rod that rotates above the wall about the point of last bonded monomer. The effective entropic potential is obtained in a similar way as in Sec. II A, i.e. by integrating over the available orientations of the rod. Even for such a simplified model, the MFPT analysis becomes algebraically difficult. In Sec. IIIB we use an interpolation by first finding analytical approximations in the two limiting case: without the rod and with a very long rigid rod, and then spanning between the two limits. The resultant detaching rate again has an acceleration factor depending on the rod length: $\left\{1 / 2+1 /\left[2+\left(3 L / \sqrt{b L_{p}}\right)\right]\right\}^{-1}$, where $b$ is the monomer size. For a long rod (yet when the condition $L<L_{p}$ still holds), this factor reduces to 2 , independently of the details of the lateral bond. In Sec. [V], we explore the full process of irreversible unbinding, and the power-law scaling of the total unbinding time with the chain length, by summing the monomer detachment time along the filament length. Trying to keep the logic and the results clear, we move complex mathematical derivations into Appendices.

\section{FLEXIBLE CHAIN}

First we consider a flexible polymer ${ }^{34}$ composed of two different regions, bonded to the substrate and freely dangling, and represent it by the ideal bead-spring model as illustrated in Fig. 11(A). The freely dangling unzipped region starts from one grafted point, and goes along the remaining polymer backbone. The monomers in this region can move around through thermal fluctuations, while respecting the hard-wall constraint. The other region consists of monomers bonded to the wall through an attractive spring potential, with its minimum sitting at the bond length $a$ away from the wall. These bonded monomers are initially equally spaced by the segment (Kuhn) length $b$ in the longitudinal direction, giving the initial fully-bonded state. For simplicity, yet capturing the physical essence, the individual bonded monomers are only allowed to move perpendicular to the wall along $z$ axis. The lateral bond with the wall is assumed broken, when the particle reaches the bond-breaking distance denoted as $c$. We focus only on the last grafted monomer that is about to detach, with the rest of the bonded monomers assumed to be immobilized in their equilibrium positions in the spring potential.

Let us estimate the average time for this last bonded monomer (initially at $z=a$ ) to reach the bond-breaking distance $c$ for the first time, and find how it changes with the length $L$ of the dangling end. In order to achieve this, the first thing is to find the 'effective potential' along the $z$ direction that this last bonded particle experiences, which consists of the harmonic potential of the bond, $k(z-a)^{2} / 2$, and an entropic effect from the interaction between the detached chain segments and the wall.

The detached chain is treated as an ideal flexible chain of $N$ springs with $(N+1)$ monomers $(L=N b)$, with the propagator function $G_{N}\left(\boldsymbol{r}, \boldsymbol{r}^{\prime}\right)$ between end positions at $\boldsymbol{r}$ and $\boldsymbol{r}^{\prime}$, described by the 'diffusion equation ${ }^{34}$, with the initial condition $G_{N}\left(\boldsymbol{r}, \boldsymbol{r}^{\prime}\right)=\delta\left(\boldsymbol{r}-\boldsymbol{r}^{\prime}\right)$ at $N=0$ :

$$
\frac{\partial G_{N}\left(\boldsymbol{r}, \boldsymbol{r}^{\prime}\right)}{\partial N}=\frac{b^{2}}{6} \nabla^{2} G_{N}\left(\boldsymbol{r}, \boldsymbol{r}^{\prime}\right) ; G_{N}\left(\boldsymbol{r}, \boldsymbol{r}^{\prime}\right) \propto e^{\frac{-3\left(\boldsymbol{r}-\boldsymbol{r}^{\prime}\right)^{2}}{2 N b^{2}}},
$$

Without the reflecting wall, Eq. (1) has a standard Gaussian solution shown on the right of the equation.

\section{A. The effective potential}

The presence of an impenetrable wall imposes a boundary condition at $z=0$. The exact form of this boundary 

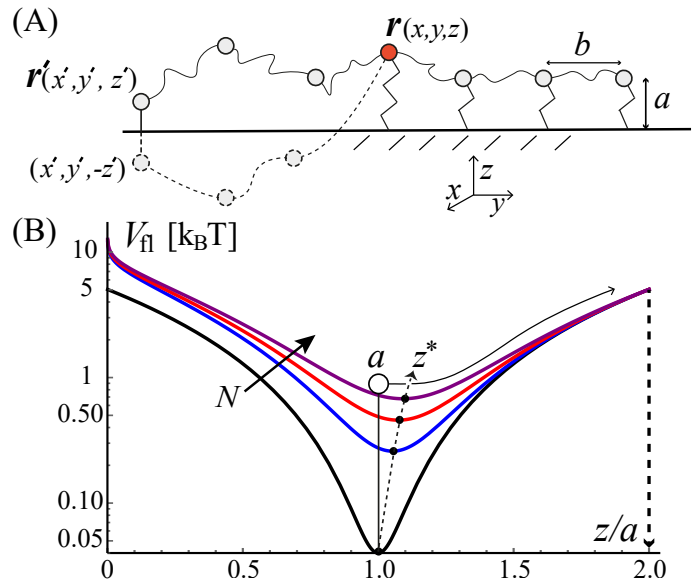

FIG. 1. (A) Cartoon of a flexible chain partially attached to a hard wall, with detached chain free to fluctuate. Our focus is on the kinetics of the tag monomer (shown in red), while the rest of the bonded monomers are immobilized. The imaged chain is shown in dashed lines, with one end imaged from $\boldsymbol{r}^{\prime}$ across the surface, yet the other end fixed at the tag monomer. Note that this imaged chain inevitably crosses the surface and is therefore a forbidden configuration that we need to exclude in calculating the number of chain configurations which obey the hard-wall boundary condition. (B) Plot of the effective potential $V_{\text {fl }}$ (logarithmic scale) against $z$, with the detached length $N=0,2,5$, and 150 (increasing along the arrow). The monomer starts from the the length $a$ and breaks the bond when reaching $z=c$ (here taken as $c=2 a$ ). The minimum of this effective potential, $z^{*}$, moves farther away from the equilibrium bond length $a$ due to the entropic repulsion, as $N$ increases.

condition is non-trivial, as simulations ${ }^{35}$ showed. Many of the important theoretical studies ${ }^{36} 38$ all used an 'exclusion' condition $G_{N}\left(\boldsymbol{r}, \boldsymbol{r}^{\prime}\right)=0$ at the wall $(z=0)$, meaning that a flexible chain strongly prefers not to touch the surface. This is an effect of configurational entropy of a long chain, examined first by DiMarzic ${ }^{39}$ by counting restricted chain configurations, and then by Edwards and Freed $\frac{40}{}$ by estimating the entropic repulsive force if the chain were pushed into a wall. It is in stark contrast with the 'zero-flux' condition on the repulsive wall that individual (colloid) particles experience.

To solve Eq. (1) with exclusion boundary condition, one can apply the 'image' principle originally introduced by Smoluchowski $\frac{41}{1 n}$ the diffusion-type problems. There is a subtlety here: the 'image' chain shares the same grafting point $\boldsymbol{r}$ with the real chain. It is only the other end of the chain that is reflected across the wall, being at the position $\left(x^{\prime}, y^{\prime},-z^{\prime}\right)$, as shown in Fig. 1(A), with the chain adopting an arbitrary configuration between these fixed ends. All such 'image' configurations are, in fact, forbidden, because it inevitably crosses the wall at a certain segment as can be seen in Fig. 1(A). Its contribution therefore needs to be subtracted from the original unconstrained propagator, giving the constrained solu- tion $G_{N}^{C}\left(\boldsymbol{r}, \boldsymbol{r}^{\prime}\right)$ as:

$$
\begin{aligned}
G_{N}^{C}\left(\boldsymbol{r}, \boldsymbol{r}^{\prime}\right) & =\left(\frac{3}{2 \pi N b^{2}}\right)^{3 / 2} \exp \left(-\frac{3\left[\left(x-x^{\prime}\right)^{2}+\left(y-y^{\prime}\right)^{2}\right]}{2 N b^{2}}\right) \\
\times & {\left[\exp \left(-\frac{3\left(z-z^{\prime}\right)^{2}}{2 N b^{2}}\right)-\exp \left(-\frac{3\left(z+z^{\prime}\right)^{2}}{2 N b^{2}}\right)\right] \cdot(2) }
\end{aligned}
$$

Since the detached chain has one freely-fluctuating end, we integrate Eq. (2) over $\boldsymbol{r}^{\prime}$, counting all the possible detached-chain configurations, while leaving $z$ as the only spatial variable for this propagator (note that the monomer is assumed to move only along $z$ direction; $x$ and $y$ do not come into play and are set to be zero):

$$
Z_{N}^{C}(z)=\int_{-\infty}^{\infty} d x^{\prime} \int_{-\infty}^{\infty} d y^{\prime} \int_{0}^{\infty} d z^{\prime} G_{N}^{C}\left(\boldsymbol{r}, \boldsymbol{r}^{\prime}\right)=\operatorname{erf}\left(\frac{z}{2 R_{g}}\right)
$$

where $\{$ erf $\}$ is the error function, and $R_{g}=\sqrt{\mathrm{Nb}^{2} / 6}$, is known as the radius of gyration. As expected, $Z_{N}^{C}$ equals to zero when the grafted monomer touches the wall, and increases when moving away from the wall. At large separation, this term reaches a plateau, entailing that an entropic repulsion is only observed near the wall. Equation (3) has been used in similar polymer systems, on different topics, all dedicated to understanding the entropic effect arising from the wal $[38 / 42 / 43]$.

The effective potential that the last bonded monomer experiences is the combination of a spring (bond) potential and an entropic contribution to free energy:

$$
V_{\mathrm{fl}}(z)=\frac{k}{2}(z-a)^{2}-k_{B} T \ln \left[\operatorname{erf}\left(\frac{z}{2 R_{g}}\right)\right] .
$$

For practical bonded polymers in biological systems, the bond length $a$ is usually of the same magnitude of the segment length $b$, while the bond-breaking distance $c$ normally sits between $2 b$ and $3 b$. The characteristic energy per monomer depends on the type of physical interaction, and could vary between $20 k_{B} T$ for stronger hydrogen bonds to around $5 k_{B} T$ for weaker hydrophobic interaction, when $k_{B} T$ is the room temperature ${ }^{13 / 44 / 45}$. For illustration, let us set $a=b=1 \mathrm{~nm}, c=2 \mathrm{~nm}$, and choose the spring constant $k$ value such that the bond energy, $k(c-a)^{2} / 2=5 k_{B} T$. Then Fig. 1(B) shows the variation of effective potential with $z$, with increasing detached length $N$. Note that in this plot we have vertically shifted the potential $V_{\mathrm{ff}}$ at different $N$ values, in order to have the same value at $z=c$ for clearer comparison. As $N$ increases, the equilibrium length of the bond, $z^{*}$, is driven away from the wall: a stronger entropic repulsion appears as the detached length increases, while at $N=0$, the effective potential is simply the spring on its own.

\section{B. Mean first-passage time and detachment rate}

The MFPT technique is a well-known method in statistical mechanics 30 , used to estimate the time for the particle to first reach the adsorbing boundary, starting from 
a specified initial position. Here, our detachment system projects into a one-dimensional problem (along the detaching direction $z$ ) for the last bonded monomer, with one adsorbing boundary at $z=c$ (after the monomer breaks the bond, we do not account for a possible rebonding as we are interested in finding the detachment rate only) and a hard wall boundary condition at $z=0$, while the monomer initially starts at $z=a$ (at the minimum of the bond potential). For this system, MFPT has a rather simple integral form:

$$
\tau=\frac{1}{D} \int_{a}^{c} d z e^{V_{\mathrm{eff}}(z) / k_{B} T}\left(\int_{0}^{z} d x e^{-V_{\mathrm{eff}}(x) / k_{B} T}\right)
$$

where $V_{\text {eff }}$ is the effective potential, and is given in Eq. (4) for a flexible chain and Eq. (13) for a semi-flexible filament. For the flexible chain, $\bar{D}$ is the bare diffusion coefficient of the single fluctuating monomer. Note that all the boundary and initial positions appear in the integral limits. When we insert Eq. (4) into the MFPT expression, the resultant integrals are difficult to calculate directly. However, we can obtain a good approximation by assuming a large spring constant $k$, which turns out to be reasonable for practical biopolymer systems with the bond energy of $5 k_{B} T$ per monomer, or above. We will show the comparison of the numerical and approximated results, after we derive them, to justify this assumption later, see Fig. 2(B).

The first/inside integral in Eq. (5) is estimated by using the saddle point approximation. The saddle point $z^{*}$ is given by the equation:

$$
\left(z^{*}-a\right)=\frac{k_{B} T \exp \left(-z^{* 2} / 4 R_{g}^{2}\right)}{\sqrt{\pi} k R_{g} \operatorname{erf}\left(z^{*} / 2 R_{g}\right)}
$$

which is not analytically solvable. But the shift $\left(z^{*}-a\right)$ becomes small when the restoring coefficient $k$ increases, which may be checked by examining the large- $R_{g}$ case and obtaining $\left(z^{*}-a\right) \propto k^{-1}$. Under the large- $k$ assumption, we take the saddle point $z^{*} \approx a$, neglect the small correction added to $k$ in the quadratic coefficient of the expansion, and further replace the upper limit of the first integral with $\infty$, as the inside exponential function decays sharply away from the saddle point. The first integral then becomes: $\operatorname{erf}\left(a / 2 R_{g}\right) \int_{0}^{\infty} e^{-k(x-a)^{2} / 2 k_{B} T} d x$. In this way, we decouple the two integrals in Eq. (5). The second integral clearly has its major contribution from around $z=c$, see Fig. 1(B). We substitute $-k_{B} T \ln \left[\operatorname{erf}\left(c / 2 R_{g}\right)\right]$ for the entropic term in the effective potential of Eq. (4), resulting in the factor $1 / \operatorname{erf}\left(c / 2 R_{g}\right)$ in the second integral. Altogether, the mean detachment rate $K_{\mathrm{fl}}$ of the $N^{\text {th }}$ segment, namely the inverse of the MFPT, takes the form:

$$
K_{\mathrm{fl}} \approx \frac{\operatorname{erf}\left(c / 2 R_{g}\right)}{\operatorname{erf}\left(a / 2 R_{g}\right)} \frac{D}{\int_{a}^{c} e^{\frac{k(z-a)^{2}}{2 k_{B} T}} d z \int_{0}^{\infty} e^{\frac{-k(x-a)^{2}}{2 k_{B} T}} d x} \approx \frac{\operatorname{erf}\left(c / 2 R_{g}\right)}{\operatorname{erf}\left(a / 2 R_{g}\right)}\left\{\frac{D(c-a) k^{3 / 2}}{\sqrt{2 \pi}\left(k_{B} T\right)^{3 / 2}\left[1+\frac{k_{B} T}{k(c-a)^{2}}\right]} e^{-k(c-a)^{2} / 2 k_{B} T}\right\}
$$

where $R_{g}=\sqrt{N b^{2} / 6}$, and the integrals in the bracket of the first line represent, in fact, is the detachment rate $K_{\mathrm{ff}}^{0}$ for the monomer with a strong spring potential of attaching to the wall, without any effect of the detached chain (at $R_{g}=0$ ). The analytic approximation of the average escape rate for such a harmonic system is well-

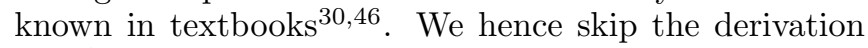
of $K_{\mathrm{f}}^{0}$ and present the final form for our detachment rate $K_{\mathrm{ff}}$ in the second line of Eq. (7).

The effect of entropic repulsion from the detached chain is manifested in the ratio of erf-functions, as a factor multiplying the 'bare' $K_{\mathrm{ff}}^{0}$. This ratio reduces to 1 when $N=0$ (i.e. without any detached chain), correctly recovering the physical picture that only spring potential matters in this extreme case. On the other hand, when the detached length $N$ grows, this ratio increases, making the detaching process faster. Yet, for a very long detached chain, it eventually reaches a plateau determined by the ratio of the breaking distance to bond length, namely, $c / a$. This acceleration pre-factor depends on the Kuhn length $b$, through the relation $R_{g}=\sqrt{N b^{2} / 6}$ : at a fixed $N$, a larger step length $b$ gives a longer detached chain $R_{g}$, thus resulting in a stronger entropic repulsion away from the wall, and accordingly a sharper increased rate. Figure 2(A) shows how $K_{\text {mathrmfl }} / K_{\text {mathrmfl }}^{0}$ ratio changes with the length of the free chain, for several values of $b$, while taking $a=1 \mathrm{~nm}$, and $c=5 \mathrm{~nm}$ to enhance the $c / a$ ratio and make the effect of $b$ more visible.

To see how the bond strength $k$ affects $K_{\mathrm{fl}}$, and whether our large- $k$ assumption leads to desirable approximated form, we plot both the numerical and approximated detachment rate (using Eqs. (5) and (7) in Fig. $2 \mathrm{~B}$ ), with $a=1 \mathrm{~nm}, b=1 \mathrm{~nm}$, and $c=2 \mathrm{~nm}$, against $k$ in the range that would give bond energy from 2.5 to $7.5 \mathrm{k}_{\mathrm{B}} \mathrm{T}$ in room temperature, with three different $N$ values. All these parameter values are in practical order of the magnitude for biological systems. Note that the diffusion coefficient $D$ of a monomer is not a relevant parameter that would cause any error, and is chosen to be 1 $\mathrm{nm} \cdot \mathrm{sec}^{-2}$ for convenience to cancel the units. The results show that $K_{\mathrm{ff}}$ decreases dramatically with $k$ as expected from the Boltzmann factor, $\operatorname{Exp}\left[-k(c-a)^{2} / 2 k_{B} T\right]$, to overcome the bond energy barrier in the Arrhenius kinetic theory, and that our approximation of Eq. (7) in Fig. 2(B) matches well with the numerical calculation 

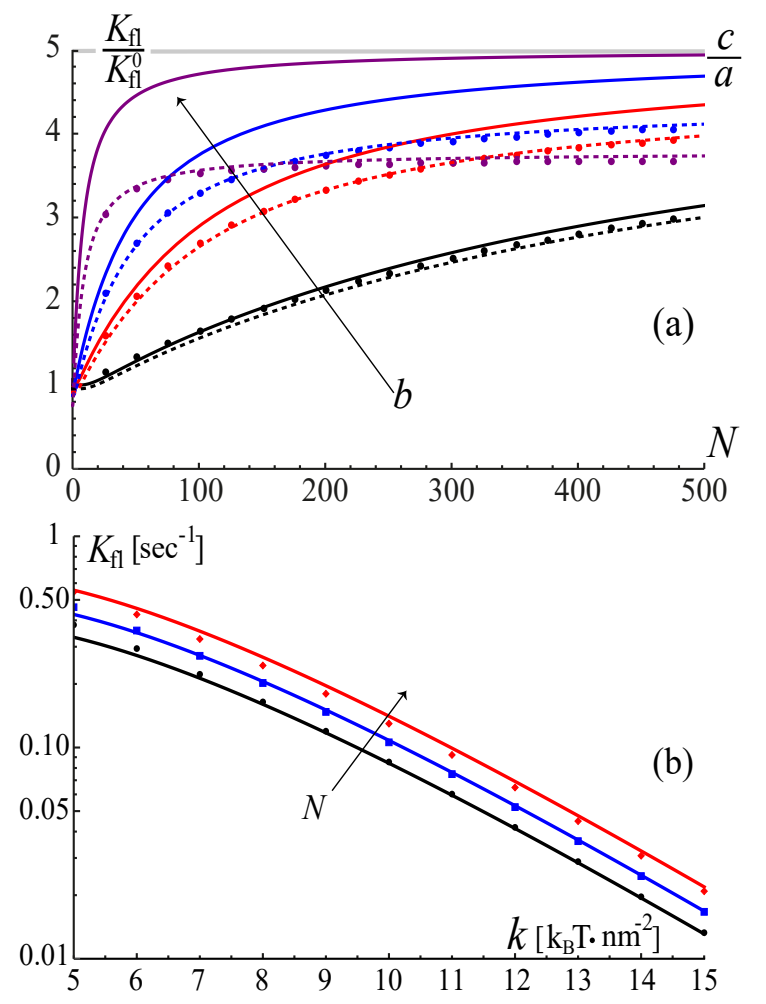

FIG. 2. (a) Acceleration pre-factor of the flexible chain, $K_{\mathrm{f}} / K_{\mathrm{fl}}^{0}$, plotted against the detached length $N$, with $b / a=$ $0.2,0.4,0.6,1.4 \mathrm{~nm}$ (increasing along the arrow). Solid lines are plotted without considering the hydrodynamic interaction between the surface and the last bonded segment. The asymptote at large $N$ is shown in grey line, giving the plateau $c / a$. The corrections due to the hydrodynamic effect, Eq. (9), are shown in dashed lines, with circles being numerical results (hydrodynamic effect included), using the same parameter values. (b) Plot of the average detachment rate $K_{\mathrm{fl}}$ (logarithmic scale) of the flexible chain against the spring constant $k$, for $N=1,3$, and 15 . Solid lines are produced using the approximated expression of Eq. (7), while markers (circles, squares and diamonds for $N=1,3$ and 15, respectively) are the corresponding numerical results estimated from Eq. (5).

(within $10 \%$ error).

Note that our assumption that the diffusion coefficient $D$ is a constant independent of the monomer position with respect to the wall is based on ignoring the volume/thickness of segments in the bead-spring model. It is well-known that particles with non-zero volume show a decreased mobility (and thus the local diffusion coefficient $D(z)$ in the vicinity of a hard wall), which is due to hydrodynamic effects of flow disruption 4750 . This effect may be important for attaching and detaching kinetics in biological systems, for example, in the shaft dynamics of a bacterium attached to the hard surface 51 . In our system, we consider only the hydrodynamic interaction between the hard wall and the last bonded segment, and neglect the hydrodynamic effect from the immobilized and other remaining monomers of the free chain, which we take to be a Rouse chain. For a spherical particle of radius $b$ (we take it of the order of the segment length), $D(z)$ has an approximate form $D_{\infty} C(z, b)$, where $D_{\infty}$ is the diffusion coefficient far away from the hard wall, and the correction factor $C(z, b)$ is given by the famous expression $\frac{47 / 49}{}$ :

$$
C(z, b) \approx \frac{6(z / b)^{2}+2 z / b}{6(z / b)^{2}+9 z / b+2},
$$

which equals to zero at $z=0$, and reaches one far away from the wall.

The mean detachment rate we evaluated in Eq. (5) needs to be modified, since the effective diffusion constant $D(z)$ is now under the outer integral over $z$, see Ref. ${ }^{29}$. The calculation is straightforward, and we plot its numerical result in Fig. 2(A) with circles, to illustrate the level of correction introduced by this hydrodynamic effect. Since the effective diffusion is reduced near the wall, the role of this hydrodynamic correction is to decrease the mean detachment rate of the last bonded monomer, although not significant for some typical values of parameters. In the same way we produced the approximate analytical factors in $K_{\mathrm{f}} / K_{\mathrm{ff}}^{0}$ in Eqs. (6) and (7), a simple approximation for the hydrodynamic correction at high lateral-bond strength $k$ takes the form:

$$
\frac{K_{\mathrm{fl}}}{K_{\mathrm{fl}}^{0}} \approx \frac{\operatorname{erf}\left(c / 2 R_{g}\right)}{\operatorname{erf}\left(a / 2 R_{g}\right)} C(c, b),
$$

which clearly shows that only the diffusion coefficient near bond-breaking distance $c$ matters, appearing as the factor $C(c, b)$. As the segment size $b$ increases, with respect to the equilibrium bond length $a$, the hydrodynamic effect becomes more prominent, cf. dashed lines in Fig. 2(A).

In summary, we exposed the origin of the entropic repulsion originating from the hard wall in Sec. IIA , and further implement MFPT method to investigate how this affects the monomer detachment rate. The result gives an acceleration ratio $\left[\operatorname{erf}\left(c / R_{g}\right) / \operatorname{erf}\left(a / R_{g}\right)\right]$ multiplying the unbinding rate of the purely spring potential case (i.e. $\left.K_{\mathrm{fl}} 0\right)$. In the long chain limit, this ratio reduces to $c / a$, merely determined by the equilibrium bond length and its breaking distance.

\section{SEMI-FLEXIBLE FILAMENT}

Semi-flexible chains are rigid polymers where monomers prefer to align linearly. This tendency is conveniently described by the famous Kratky-Porod discrete model or the continuous Worm-like chain mode ${ }^{33 \mid 34}$, through the bending energy:

$$
H_{b}=\sum_{i=0}^{N} \frac{\kappa}{2 b}\left(1-\cos \theta_{i}\right) \sim \int_{0}^{N b} \frac{\kappa}{2}\left[\frac{d^{2} \boldsymbol{r}_{(s)}}{d s^{2}}\right]^{2} d s,
$$

where $\theta_{i}$ is the bond angle between bond vectors of $i$-th and $(i+1)$-th monomers (the tangent vector for the $i$ th monomer is denoted as $\left.\boldsymbol{t}_{i}\right), \kappa$ is the bending rigidity, 


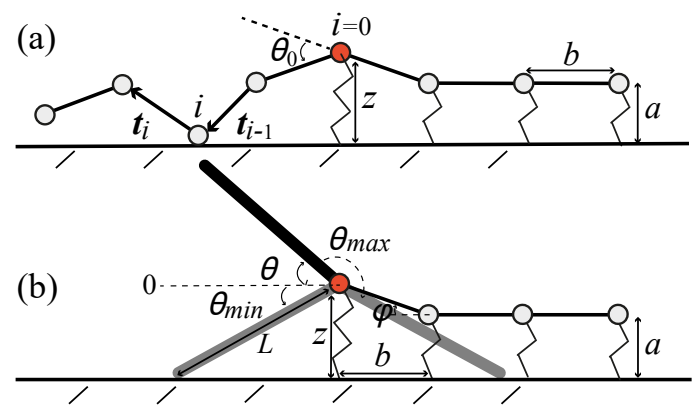

FIG. 3. (a) A discrete presentation of a semi-flexible filament above the wall. A monomer on the wall is particularly shown to illustrate the hard wall boundary conditions on the bond vector $\boldsymbol{t}$. (b) Rod-like description used in our model. $\theta_{\max }$ and $\theta_{\min }$ are the maximum and minimum of the available rotation angle $\theta$ about the tag monomer, respectively. $\phi$ is the bending angle between the second and third bonded monomer.

$b$ is the internal bond length between two neighboring monomers, see Fig. 3(A). In the continuous version, $\boldsymbol{r}$ is the position of the chain element, as a function the arc length $s$ along the semi-flexible filament. The second derivative of $\boldsymbol{r}$ is the local curvature along the chain.

The persistence length $L_{p}$ is defined as the ratio $\kappa / k_{B} T$, and is the length scale for the chain to roughly retain its linear shape without significant bending fluctuation. When the chain length, $L \equiv N b$, is much longer than $L_{p}$, a behavior similar to the flexible chain is expected. Hence, we will only focus on the regime $L<L_{p}$, as the opposite limit to the previous section. We ignore any possible torsional effect along the chain, and use a discrete model of the two-dimensional system, as depicted in Fig. 3(A). As in the flexible chain case, the bonded monomers are all assumed to be immobilized in their equilibrium position of the spring potential (at $z=a$ ), except for the last bonded monomer that is considered as the grafting point of the remaining free chain. Both the dangling chain end and the last bonded monomer are subject to thermal fluctuations.

\section{A. The effective potential of a rod-like model}

The presence of a wall poses a different boundary condition on its surface, compared with the flexible chain, where monomers are strongly repelled away and cannot reside against the wall due to great loss of configurational entropy. The stiffness of semi-flexible chain can overcome this entropic loss and force monomers to lean on the wall. To put it differently, if the $i$-th monomer rests on the wall, the tangent vectors $\boldsymbol{t}_{i-1}$ and $\boldsymbol{t}_{i}$ must point downward and upward, respectively, see Fig. 3(A) for illustration.

This wall boundary condition is non-trivial to implement, when one tries to solve the propagator equation for the partition function of the chain. Under a subtle as- sumption of a long semi-flexible chain with small lateral deflection (yet still in the regime $L<L_{p}$ ), the partition function for a chain with one fluctuating end and the other end grafted with specified position and bond orientation above the wall has been obtained as a product of $z^{1 / 6}$ and a complicated hypergeometric function that accounts for the orientation effect 26128 . However, the information of the chain length has been lost or becomes simply a free energy shift that plays no effect in the shape of the effective potential, making it unsuitable in our purpose (which is to investigate how this length affects the unzipping time).

Instead of solving the propagator for the chain, we treat the detached chain as a rigid rod, free to rotate above the wall, as our interest lies in the regime $L<L_{p}$. This rod-like system has only two bending energy contributions. One is due to the bending angle $\theta$ between the rod and the first polymer tangent in the bonded area, while the other is the angle $\phi$ between the first and second bonds therein, as shown in Fig. 3(B). Without the dangling rod, the chain Hamiltonian solely depends on the bent angle $\phi$. Note that this angle $\phi$ is essentially a function of unbinding coordinate $z$, described by the relation: $\sin \phi=(z-a) / \sqrt{b^{2}+(z-a)^{2}}$.

The hard-wall boundary condition in this rod-like model becomes a constraint on the range of the available rotation angle $\theta$. For $L>c$ ( $c$ is the bond-breaking length), this constraint can be readily obtained from triangular geometry, when this rod rotates clockwise and counter-clockwise to hit the wall, see Fig. 3(B): $\theta_{\min }=$ $\cos ^{-1}(z / L)-\pi / 2$, and $\theta_{\max }=-\cos ^{-1}(z / L)+3 \pi / 2$. For $L<c$, this has to be discussed in a piecewise way in two regimes $z>L$ and $z \leq L$, yet this extra complexity may be unnecessary, as one realizes that $c$ is usually only two or three monomers long and this $L<c$ regime is indeed very narrow over the entire course of the full detachment. Alternatively, we can first find the effective potential of $L=0$ and $L>c$ cases, then use them to estimate the detachment rate, and finally interpolate to cover the $L<c$ regime.

Without the rod (that is, the free dangling part), the effective potential is the sum of the spring potential plus the bending energy, $-\kappa(\cos \phi-1) / 2 b$ :

$$
V_{\mathrm{sf}}^{0}=\frac{k}{2}(z-a)^{2}-\frac{\kappa}{2 b}\left[\frac{b}{\sqrt{b^{2}+(z-a)^{2}}}-1\right] .
$$

When $z=a$, the bending energy in $V_{\mathrm{sf}}^{0}$ is zero, whereas any $z$-shift from $a$ creates non-zero bending energy, in addition to the spring potential.

For the rod with $\theta$ angle, the bending energy is: $-\kappa[\cos (\phi-\theta)+\cos \phi-2] / 2 b$. The partition function is found by integrating all available rotations: $\int_{\theta_{\min }}^{\theta_{\max }} \exp \left\{\kappa[\cos (\phi-\theta)+\cos \phi-2] / 2 b k_{B} T\right\} d \theta$. Assuming a large $\kappa$, we expect the bending energy to be lowest around $\theta=\phi$ (prefer to align linearly), and hence implement Gaussian approximation around this point. The integrand in this partition function shall decay sharply, 
under this large $\kappa$ assumption. Meanwhile, we notice that the angle $\theta_{\max }$ has the rod rotate almost into the opposite alignment direction, far enough away from the preferred angle $\phi$, so to be replaced with $\infty$ in the integral limit. On the other side, $\theta_{\min }$ may still be close to $\phi$ to cover the major contributions of this decaying integrand, thus remains in its original form, and is the only parameter that includes the effect of the rod length $L$.
Altogether, the partition function $G_{\mathrm{sf}}$ is:

$$
G_{\mathrm{sf}} \sim \int_{\theta_{\min }}^{\infty} \exp \left\{\frac{L_{p}}{2 b}\left[\cos \phi-\frac{1}{2}(\theta-\phi)^{2}-1\right]\right\} d \theta .
$$

The $G_{\text {sf }}$ integral is straightforward, and the corresponding effective potential is a spring potential plus the rod repulsion term $\left(-k_{B} T \ln G_{\mathrm{sf}}\right)$ :

$$
\begin{aligned}
V_{\mathrm{sf}} & =\frac{k}{2}(z-a)^{2}-k_{B} T\left\{\ln \sqrt{\frac{\pi b}{L_{p}}}+\frac{L_{p}}{2 b}(\cos \phi-1)+\ln \left[1+\operatorname{erf}\left(\frac{1}{2} \sqrt{\frac{L_{p}}{b}}\left(\phi-\theta_{\text {min }}\right)\right)\right]\right\} \\
& \approx V_{\mathrm{sf}}^{0}(z)-k_{B} T\left\{\ln \left[1+\operatorname{erf}\left(\frac{1}{2} \sqrt{\frac{L_{p}}{b}}\left(\phi-\theta_{\text {min }}\right)\right)\right]-\ln 2\right\},
\end{aligned}
$$

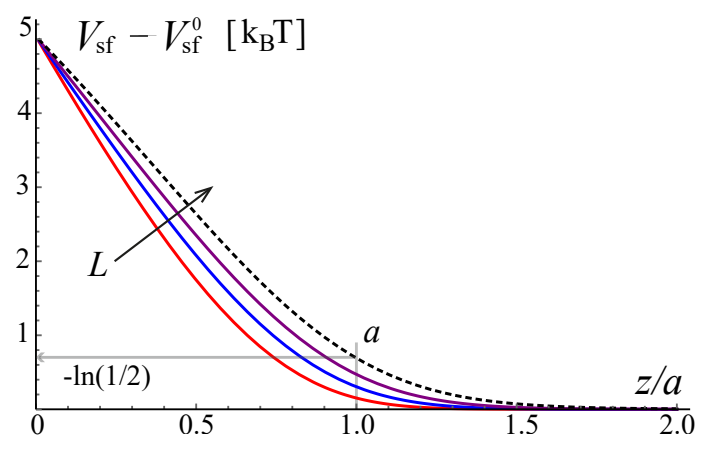

FIG. 4. Entropic potential of repulsion due to the constrained rotation above the hard wall, plotted against the unzipping coordinate $z$ with increasing rod length: $L=3,5$ and $10 \mathrm{~nm}$ along the arrow, while setting $a=1, b=1, c=2$ and $L_{p}=$ $20 b$. The asymptote of a long rod is shown in dashed line, which intersects with $z=a$ roughly at $-\ln (1 / 2)$, contributing to a decrease in effective potential barrier of detachment.

where $V_{\mathrm{sf}}^{0}$ is the effective potential without the free dangling rod, Eq. (11), and the angle $\phi$ contains an additional $z$-dependence via $\cos \phi=b / \sqrt{b^{2}+(z-a)^{2}}$. The constant terms are discarded in the second line of Eq. (13). We also note that when $z$ increases, the angle $\phi$ also grows, making the erf-term approach 1 . We hence add the shift $(-\ln 2)$, so that $V_{\mathrm{sf}}$ overlaps with $V_{\mathrm{sf}}^{0}$ as $z$ reaches the bond-breaking length $c$, which is more convenient to compare the potential profiles of varied $L$ values.

The interplay between the rod and wall is manifested in the $-k_{B} T\{\ln [1+\operatorname{erf}(\cdots)]-\ln 2\}$ term, which we plot by setting $a=1, b=1, c=2$ and $L_{p}=20 \mathrm{~nm}$ in Fig. 4 . with varied rod lengths $L$. This entropic repulsion increases when $z$ approaches zero, yet not goes to infinity as in the flexible chain case, because semi-flexible filament allows monomers to rest on the wall and only puts constraint on the bond-orientation. On the other hand, when the monomer moves far away from $a$, the rotation range of angle $\theta$ roughly recovers the partition function $G_{\mathrm{sf}}$ without the constraint, that is, Eq. $(12)$ with the integral limits $\pm \infty$. This causes the repulsion free energy curve reduced to zero as $z$ increases, regardless of $L$. In fact, at around $z=c$, this repulsion almost vanishes in Fig. 4

A cross-over regime between these two extremes $(z$ close to, and very far from the wall) appears around $z=a$. It is interesting to note that a long rod at $z=a$ can rotate only upward (thus inserting $\phi=0$ and $\theta_{\min }=0$ in Eq. (12)). This approximately gives half of $G_{\mathrm{sf}}$ of less constrained case at $z=c$, thus resulting in a free energy increase of $-k_{B} T \ln (1 / 2)$ around $z=a$ shown in Fig. 4. In turn, the detachment potential barrier, with the effective potential well sitting roughly around $z=a$ and a zero repulsion at $z=c$ (as explained above), decreases roughly by $-k_{B} T \ln (1 / 2)$ as well. We will see this $(1 / 2)$-factor contribute to the numerical factor of 2 in the mean detachment rate in the next section.

\section{B. Mean detachment rate}

The mean detachment time without the rod in the semi-flexible chain, is calculated by inserting the effective potential Eq. (11) into Eq. (5). As stated previously, the spring constant $k$ for practical systems can be safely assumed to be large. We can therefore apply the saddle point approximation for the inside integral of Eq. (5), and replace its limits with $\pm \infty$, which rules out the $z$-dependence (the same logic as we used with the flexible chain in Sec. IIB. We recognize that the second integral has its main contribution around $z=c$, and hence Taylor-expand the exponent of the inside integrand around this point to the first order, approximating it as an easy-to-integrate exponential which decays fast when 
$z$ decreases from $c$. All these derivation details are moved to A. The final result gives the expression for $K_{\mathrm{sf}}^{0}$, which is the average rate of breaking the bond of the monomer without the dangling rod:

$$
K_{\mathrm{sf}}^{0} \approx \frac{D(c-a)\left[\frac{k}{k_{B} T}+\frac{L_{p}}{2\left(b^{2}+(c-a)^{2}\right)^{3 / 2}}\right] \sqrt{\frac{2 k}{k_{B} T}+\frac{L_{p}}{b^{3}}}}{2 \sqrt{\pi} \exp \left[\frac{k}{2 k_{B} T}(c-a)^{2}+\frac{L_{p}}{2 b}\left(1-\frac{b}{\sqrt{b^{2}+(c-a)^{2}}}\right)\right]} .
$$

The bending energy, embedded in the expression for $L_{p}$, appears together with the bond energy as the combined energy barrier to overcome for breaking the bond. It appears in the activation exponential factor, while the details of the potential shape, around the potential well and around the bond-breaking distance, are reflected in the numerator. For $L_{p}=0, K_{\mathrm{sf}}^{0}$ reduces to the spring potential case, in agreement with the expression for $K_{\mathrm{f}}^{0}$, i.e. the term inside the curly bracket in Eq. (7).

When we put effective potential of the rod-like case, $V_{\text {sf }}(z)$ of Eq. (13), into the MFPT integral of Eq. (5) to calculate the detachment rate, the algebra becomes quite complicated, and the approximation we use before does not lead to a clear analytical expression. Instead, we first find the average detachment rate for the limiting case of a very long rod, and then combine it with the rod-free result $K_{\mathrm{sf}}^{0}$, and the numerical calculation of the finite rod case, in order to propose a good interpolation.

For a very long rod, the angle $\theta_{\min }$ in Eq. (13) becomes zero. By using the resultant effective potential and following the similar procedures (see B for derivation), we find the simple approximation for the mean detachment rate in the limit of a long rod: $K_{\mathrm{sf}}^{\infty} \approx 2 K_{\mathrm{sf}}^{0}$ (within $10 \%$ error compared with numerical results). Perhaps, this is not a too surprising, as we already explained below Eq. 13), and illustrated in Fig. 4, that a long rod roughly lowers the potential barrier by $-k_{B} T \ln (1 / 2)$, due to constrained rotation range around $z=a$ (ruling out downward half-plane rotations).

The next task is to find an interpolation factor that reduces to one when the rod length $L=0$, and reaches 2 as $L \rightarrow \infty$, by observing how the numerical results of the average detachment rate of a finite rod behaves. This factor is not an exponential-related function as one would guess at first sight, but is better fitted by the inverse of a shifted scaling function, giving the mean detachment rate of a finite rod as:

$$
K_{\mathrm{sf}} \approx\left(\frac{1}{2}+\frac{1}{2+3 L / \sqrt{b L_{p}}}\right)^{-1} K_{\mathrm{sf}}^{0} .
$$

This interpolation is within $10 \%$ error over a wide range of parameter space, and even more accurate for the range of practical biological bonded polymers, when compared with the numerical results (see C for this comparison). The halfway length can be defined as the rod length $L^{*}$ that makes this pre-factor mid-way between 1 and 2 , giving $L^{*}=2 \sqrt{L_{p} b} / 3$. A more rigid chain has a longer

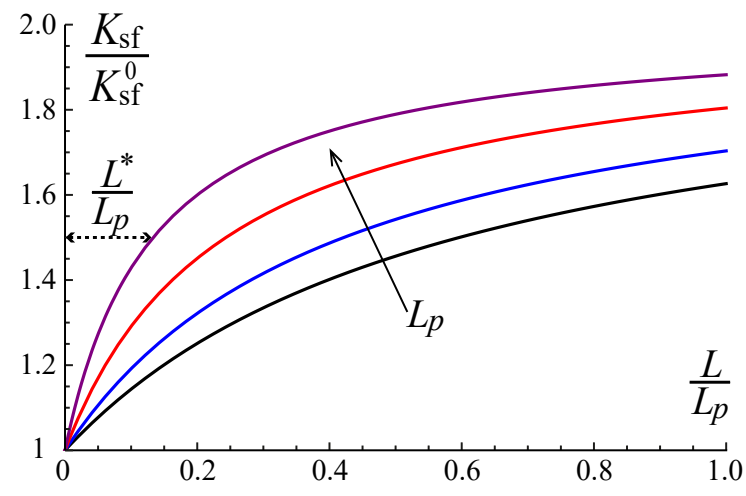

FIG. 5. Acceleration pre-factor of the rod-like model, $K_{\mathrm{sf}} / K_{\mathrm{sf}}^{0}$, plotted against the scaled rod length $L / L_{p}$, for $L_{p}=5 b, 10 b, 30 b$ and $100 b$. (increasing along the arrow).

halfway length, and the mean detachment rate in this case reaches the plateau of $K_{\mathrm{sf}}{ }^{0}$ more slowly while the dangling rod length increases. This may be understood if we realize that the angle $\theta_{c r}$ to include the major region of the peak of $G_{\mathrm{sf}}$ shrinks, as $L_{p}$ increases, making this peak sharper. Accordingly, $\theta_{\min }$ requires a longer $L$ to shrinks more pronouncedly, in order to have $\left|\theta_{\text {min }}\right|<\left|\theta_{c r}\right|$ and subsequently a reduced $G_{\mathrm{sf}}$ value that creates the entropic repulsion in the effective potential.

Although it would be desirable to also include the hydrodynamic effect near the hard wall as in the flexible chain, finding the diffusion coefficient of the rod end moving along the $z$ direction is not straightforward, due to its coupling with both translational and rotational diffusion coefficients in the geometry of a rod with the hinged end. Careful investigation of the correction factor of such effect in $D$ goes beyond the focus of this paper, and will not be address it here.

The rod-like model is valid in the regime where $L<L_{p}$. For a less rigid rod, when $L=L_{p}$, the acceleration factor of Eq. (15) may have not yet reached its 2-limit. We plot this pre-factor, $K_{\mathrm{sf}} / K_{\mathrm{sf}}^{0}$, against the scaled rod length $L / L_{p}$, with $b=1 \mathrm{~nm}$ and several $L_{p}$ values in Fig. 5 . Therein, the scaled halfway length, $L^{*} / L_{p}$, is longer for a smaller $L_{p}$, consequently producing a smaller $\left(K_{\mathrm{sf}} / K_{\mathrm{sf}}^{0}\right)$ at the limit $L=L_{p}$.

\section{DISCUSSION AND CONCLUSIONS}

We now consider the case of an irreversible one-byone detaching process for a polymer of $N$ segments from the end. Although we are aware that the exclusion of re-combination is not realistic in biological systems such as DNA-zippers, our aim here is merely to explore how the entropic acceleration of the detaching rate due to the free dangling chain could possibly affect the $N$-scaling of the total detaching time. The total/cumulative detaching time (excluding the possibility of recombination) can be obtained by integrating the inverse of Eqs. (7) and 
15. over the number of detached monomers $N$ (equal to $L / b)$. For flexible polymers, the inverse of Eq. $(7)$ is not analytically integrable. We therefore first numerically estimate the non-dimensional cumulative unbinding time $\tau_{\mathrm{fl}}^{\text {tot }}$ to observe its $N$-scaling, and then propose a possible approximation for $\tau_{\mathrm{fl}}^{\text {tot }}$ later. Figure 6 plots the nondimensional total unbinding time $\left(\tau_{\mathrm{f}}^{\text {tot }}\right)$ as a function of $N$ on the log-log scale, and shows that the curve switches from the initial linear scaling law, $N$, to the shifted linear scaling, $(a N / c)$, when the total length of the chain increases. The lower boundary of the cross-over regime can be readily obtained by Taylor-expanding the acceleration ratio against $N$, and assuming that when the ratio of the first correction term to the zeroth term reaches $m$, the deviation of the curve starts to become important. For the upper boundary, we solve the approximate $N$-value that gives the acceleration ratio as $(1+p) a / c$, where $p$ measures how far the curve is away from the asymptotic plateau, $(a / c)$. These give the expressions for the boundaries as: $3 \pi m^{2} a^{2} / 2 b^{2}$ (lower) and $\left[c^{2}(1+p)-a^{2}\right] / 2 b^{2} p$ (upper), and we manually choose $m=0.5$ and $p=0.02$ to qualitatively cover the crossover regime. Note that the lower boundary is insensitive to $c$, due to the fact that $a \leq c$ and hence the variation of $c$ inside $\operatorname{erf}\left(c / 2 R_{g}\right)$ becomes less significant than the $a$-value inside $\operatorname{erf}\left(a / 2 R_{g}\right)$, when $R_{g}$ is a small value and erf-functions almost reach the plateau.

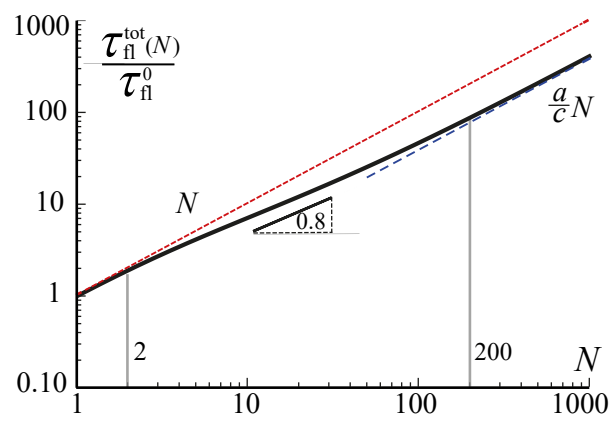

FIG. 6. Non-dimensional total unbinding time for the flexible chain plotted against $N$, with $b=1, a=1.2$, and $c=3$ $\mathrm{nm}$ as an illustrative example. The cross-over regime is the region confined by two grey lines at $N=2$ and 200, where the 'apparent' $N$-scaling changes to $\propto N^{0.8}$. The dashed lines are the asymptotic expressions at small and large $N$.

The range of this crossover extends roughly from 2 to a few hundreds of monomers, which covers the commonlyseen polymer sizes in biological systems. Moreover, the $N$-scaling in this crossover regime is changed to an apparent $N^{0.8}$. We note that previous study on desorption kinetics of the flexible polymer chains from the surface, gives this $N$-scaling exponent $\approx 2$, Ref. ${ }^{7}$. This discrepancy originates from the different configuration assumed for the zipped (bonded) state. In our model, we assume that monomers are detaching in an orderly way on the premise that the bonding is strong and only the detachment at the end of bonded monomers matters, whereas Ref. ${ }^{7}$ uses a randomly-bonded configuration for the bonded region (it does not assume a fixed number of bonded sites) and, importantly, the chain length between two neighboring bonded monomers is assumed to be larger than $b N^{3 / 5}$, so that they can apply the mesoscopic blob model to describe the chain configuration ${ }^{52}$. These blobs in the bonded region provide additional configurational entropy that would more or less stabilize the zipped state, which one expects to slow the entire unbinding process.

In contrast, our model does not allow any distance between two bonded sites (we assume it to be the Kuhn length $b$ and not much stretchable). In fact, in biological zippers such as microtubules and DNA, the lateral bonds between two parallel filaments are not really random (the bonding sites need to match the correct sites to form bonds). The simulation of the unzipping of Y-shaped DNA which excluded the effect of DNA-looping showed that this $N$-scaling of the unbinding time is $\approx 15$, which corresponds to the case where no hard wall appears, and explains that the difference in configurations assumed for zipped states could cause the different $N$-scaling in kinetics. The decreased exponent in $N$-scaling in our model originates from the switch between the two linear scalings is driven by the entropic repulsion away from the hard wall.

The approximated formula for $\tau_{\mathrm{fl}}^{\text {tot }}(N)$ can be obtained by separating the original integral over $N$ into two smaller integrals in the small- and large- $N$ regimes, respectively, and utilising the first term of Bürmann series for erf-function: $\operatorname{erf}(x) \approx\left[1-\exp \left(-x^{2}\right)\right]^{1 / 2}$, followed by Taylor-expansion of the resultant function inside the square root in the large- $N$ regime. This gives the nondimensional cumulative unbinding time approximately as:

$$
\frac{\tau_{\mathrm{fl}}^{\mathrm{tot}}}{\tau_{\mathrm{fl}}^{0}} \approx 1+\frac{a}{c}(N-1)+\frac{3 a c}{8 b^{2}}\left(1-\frac{a^{2}}{c^{2}}\right) \ln \left(1+\frac{N-1}{\nu}\right), \text { where } \nu=\frac{\frac{1}{b}\left(8 b^{2}+3 c^{2}-3 a^{2}\right)+\sqrt{4 b^{2}+3 a^{2}-3 c^{2}}}{8 b+2\left(8 b^{2}+3 c^{2}-3 a^{2}\right) / \sqrt{4 b^{2}+3 c^{2}-3 a^{2}}}
$$

$\tau_{\mathrm{f}}^{0}$ is the unbinding time required when without free dangling chain, namely $1 / K_{\mathrm{f}}^{0}$ defined below Eq. (7). The first two terms of Eq. 16 give the large- $N$ asymptote $(a N / c)$, and the last term is the correction for small $N$. The parameter $\nu$ represents the length of the free dangling end governing the sharpness of the transition be- 
tween two regimes in Fig. 6, it depends on the geometric parameters of the bond: $a$ and $c$, and Kuhn length of the chain $b$. Yet, $\nu$ only varies between 1.6 and 4.5 for $c$ between $2 b$ and $5 b$, with $a \approx b$, qualitatively consistent with the lower boundary of cross-over we proposed earlier (both would give small numbers for the occurrence of cross-over). The characteristic length $\ell_{\mathrm{fl}}$ for the crossover may be estimated by finding the intersection of $\tau_{\mathrm{fl}}^{\text {tot }}$ with the linear function that has the mean slope of these two asymptotic $N$-scalings (so that this function sits right in the mid-region in between them). Mathematically, it is to solve the $N$-value satisfying the equation: $\tau_{\mathrm{fl}}^{\text {tot }}=(1+a / c) N \tau_{\mathrm{fl}}^{0} / 2$. Although the exact analytical solution for this equation is not available, a good approximation, valid over the range of practical parameter values for biological zipped filaments, takes the form:

$$
\ell_{\mathrm{fl}} \approx 2+\frac{9 a c}{8 b^{2}}\left(1+\frac{a}{c}\right)
$$

This characteristic length $\ell_{\mathrm{fl}}$ decreases as the Kuhn length $b$ increases, because as shown in Fig. 2(A), a larger $b$ shrinks the decay length for the acceleration ratio for the unbinding time, making it approach the large- $N$ asymptote faster (and also the same for the cumulative unbinding time). On the other hand, $\ell_{\mathrm{fl}}$ increases as $a$ and $c$ increases, with quadratic and linear dependence, respectively.

For semi-flexible filaments in our model, the nondimensional total unbinding time is obtained by directly integrating the acceleration pre-factor in Eq. (15) over the filament length $N$ (use the relation $L=N b$ ), giving:

$$
\frac{\tau_{\mathrm{sf}}^{\mathrm{tot}}}{\tau_{\mathrm{sf}}^{0}} \approx \frac{N}{2}+\frac{1}{3} \sqrt{\frac{L_{p}}{b}} \ln \left(1+\frac{3}{2} \sqrt{\frac{b}{L_{p}}} N\right) .
$$

$\tau_{\mathrm{sf}}^{0}$ is defined as $1 / K_{\mathrm{sf}}^{0}$ of Eq. (14). A switching behavior similar to Fig. 6 for the flexible chain also emerges. However, the change of the 'apparent' scaling exponent in the cross-over regime is not as pronounced as for the flexible chain, with this exponent being roughly 0.9 (probably due to a slower decay of the acceleration ratio in unbinding time and a smaller asymptotic value in the long chain limit, compared with the flexible chain). The characteristic length $\ell_{\text {sf }}$ for this cross-over of two asymptotic $N$-scalings may be obtained by solving the equation $\tau_{\mathrm{sf}}^{\mathrm{tot}}=(1+1 / 2) N \tau_{\mathrm{sf}}^{0} / 2$, which gives:

$$
\ell_{\mathrm{sf}} \approx 1.675 \sqrt{\frac{L_{p}}{b}} .
$$

Equation (19) indicates a longer $\ell_{\mathrm{sf}}$-length for a more rigid filament. It is not surprising, as we already saw the same tendency in the acceleration ratio $\left(K_{\mathrm{sf}} / K_{\mathrm{sf}}^{0}\right)$, see Eq. 15. We could use the similar procedures to define the lower boundary of the cross-over, by finding the $N$ value at the intersection of $\tau_{\mathrm{sf}}^{\text {tot }}$ with $0.9 N \tau_{\mathrm{sf}}^{0}$ (the number 0.9 is chosen to give $10 \%$ deviation from asymptote at small $N$ ), and we obtain the expression: $0.36 \sqrt{L_{p}} / \sqrt{b}$.
Although this boundary grows with increased $L_{p}$, its value changes slowly from 1 to 5 , when $L_{p}$ increases from $10 b$ to $500 b$. On the other hand, at $L=L_{p}$, the unbinding time $\tau_{\mathrm{sf}}$ for the last bonded monomer with a rigid rod, namely $1 / K_{\mathrm{sf}}$ of Eq. (15) is about to approach the asymptote of a long rod. This $L$-point can therefore be treated as the upper boundary for the cross-over regime. But note that due to our rod assumption (requiring $L \leq L_{p}$ ), the non-dimensional total unbinding time never fully collapses into the asymptote $L / 2 b$ in the long rod limit. This phenomenon becomes more recognizable in less stiff filaments (a similar tendency in the acceleration ratio $\left.K_{\mathrm{sf}} / K_{\mathrm{sf}}^{0}\right)$.

In this paper, we apply the MFPT method to calculate the step-by-step detachment kinetic rate, by using a picture of a polymer attached to a hard-wall substrate, and study how the entropic repulsion from this wall constraint could affect the average time of monomer unbinding as the free dangling length grows. We find that for a flexible polymer, the mean rate of monomer unbinding is enhanced by a ratio $\left[\operatorname{erf}\left(c / 2 R_{g}\right) / \operatorname{erf}\left(a / 2 R_{g}\right)\right]$, depending on the lateral-bond details and the radius of gyration of the free dangling chain, $R_{g}$. This ratio approaches $(c / a)$, as $R_{g}$ increases. For a semi-flexible filament detaching from the substrate, we explore the regime where the free unbound length $L$ is smaller than the persistence length $L_{p}$, and present it as a rigid rod rotating about the last bonded monomer above a wall. We also find an acceleration factor approximately described by the expression: $\left[1 / 2+1 /\left(2+3 L / \sqrt{L_{p} b}\right)\right]^{-1}$, which reduces to 2 for a long rod, reflecting the fact that only half of the rotation angles are available due to the wall constraint. The increase length of this ratio is proportional to $\sqrt{L_{p} b}$. However, note that the validity of our rod-like model only holds when the condition $L \leq L_{p}$ is satisfied. The estimated acceleration ratio at $L=L_{p}$ for less stiff filaments does not really reach the asymptotic value 2 , but as the bending rigidity increases, this ratio gradually approaches this value.

Semi-flexible filament in the regime $L \gg L_{p}$ is similar to a flexible polymer, and is expected to recover the same acceleration factor as in the flexible chain case, with the Kuhn length being roughly of the $L_{p}$-magnitude. The cross-over from the rod-like model to flexible chain description is non-trivial, in the sense that the acceleration ratio first decreases to a value somewhat near 2 at $L=L_{p}$, then has to increase a bit due to a few more rotational angles becoming available along the increasingly bendable free filament, which eases the entropic repulsion, and then eventually decrease to the long-flexible chain limit $(c / a)$.

For both flexible chain and semi-flexible filament in an irreversible unbinding process, the cumulative/total detaching time shows a cross-over between two linear asymptotic $N$-scalings. The apparent $N$-scaling exponent in this cross-over regime is decreased to smaller than one, due to the entropic driving force. In this paper, we only studied the entropic driving force in the detaching 
process. In fact, some other important driving factors for unbinding include lateral pulling force at the polymer end (in DNA), and a natural local curvature along the filament contour length (in microtubules). The lateral pulling force will add a factor $\operatorname{Exp}\left(f z^{\prime} / k_{B} T\right)$ to the partition function of Eq. (2) for the flexible chain, which later needs to be integrated over $z^{\prime}$, while an energy term $-f L \sin \theta$ to our effective potential of Eq. (13) for the semi-flexible filament. On the other side, the natural curvature will apparently breaks the geometry of a straight rod, and requires a new geometry model to deal with. These topics will be addressed in our future work.

\section{ACKNOWLEDGEMENTS}

This work is funded by the Theory of Condensed Matter Critical Mass Grant from EPSRC (EP/J017639).

${ }^{1}$ A. Halperin, "The desorption of polymeric surfactantsA Kramers rate theory," Europhys. Lett. 8, 351-356 (1989)

${ }^{2}$ J. F. Douglas, H. E. Johnson, and S. Granick, "A simple kinetic model of polymer adsorption and desorption," Science 262, 2010-2012 (1993)

${ }^{3}$ A. Milchev and K. Binder, "Static and dynamic properties of adsorbed chains at surfaces: Monte Carlo simulation of a beadspring model," Macromolecules 29, 343-354 (1996)

${ }^{4}$ R. Lipowsky, "Flexible membranes with anchored polymers," Colloids Surf. A 128, 255-264 (1997)

${ }^{5}$ R. Descas, J.-U. Sommer, and A. Blumen, "Static and dynamic properties of tethered chains at adsorbing surfaces: a Monte Carlo study," J. Chem. Phys. 120, 8831-8840 (2004)

${ }^{6}$ N. Källrot and P. Linse, "Dynamic study of single-chain adsorption and desorption," Macromolecules 40, 4669-4679 (2007)

${ }^{7}$ J. Paturej, A. Milchev, V. G. Rostiashvili, and T. A. Vilgis, "Polymer detachment kinetics from adsorbing surface: theory, simulation and similarity to infiltration into porous medium," Macromolecules 45, 4371-4380 (2012)

${ }^{8}$ P. M. Claesson, E. Blomberg, J. C. Fröberg, T. Nylander and T. Arnebrant, "Protein interactions at solid surfaces," Adv. Colloid Interface Sci. 57, 161-227(1995)

${ }^{9}$ M. Aliee and A. Najafi, "Mechanical properties of an adsorbed elastic polymer in contact with a rigid membrane," Phys. Rev. E 78, $051802(2008)$

${ }^{10} \mathrm{P}$. T. Tran, P. Joshi, and E. D. Salmon, "How tubulin subunits are lost from the shortening ends of microtubules," J. Struct. Biol. 118, 107-118 (1997)

${ }^{11}$ I. M. Jánosi, D. Chrétien, and H. Flyvbjerg, "Structural microtubule cap: stability, catastrophe, rescue, and third state," Biophys. J. 83, 1317-1330 (2002)

${ }^{12} \mathrm{~J}$. Howard and A. A. Hyman, "Dynamics and mechanics of the microtubule plus end," Nature 422, 753-758 (2003)

${ }^{13} \mathrm{~S}$. Cocco, R. Monasson, and J. F. Marko, "Force and kinetic barriers to unzipping of the DNA double helix," Proc. Natl. Acad. Sci. U.S.A. 98, 8608-8613 (2001)

${ }^{14}$ S. Cocco, J. F. Marko, and R. Monasson, "Theoretical models for single-molecule DNA and RNA experiments: from elasticity to unzipping," C. R. Phys. 3, 569-584 (2002)

${ }^{15}$ F. Ritort, "Single-molecule experiments in biological physics: methods and applications," J. Phys. Condens. Matter 18, R531R538 (2006)

${ }^{10}$ J. W. J. Kerssemakers, E. L. Munteanu, L. Laan, T. L. Noetzel, M. E. Janson, and M. Dogterom, "Assembly dynamics of microtubules at molecular resolution," Nature 442, 709-712 (2006).
${ }^{17}$ N. Singh and Y. Singh, "Statistical theory of force-induced unzipping of DNA," Eur. Phys. J. E 17, 7-19 (2005)

${ }^{18} \mathrm{~J}$. Kierfeld, "Force-induced desorption and unzipping of semiflexible polymers," Phys. Rev. Lett. 97, 058302 (2006)

${ }^{19}$ A. Mossa, M. Manosas, N. Forns, J. M. Huguet, and F. Ritort, "Dynamic force spectroscopy of DNA hairpins: I. force kinetics and free energy landscapes," J. Stat. Mech. Theory Exp. 2009, P02060 (2009)

${ }^{20}$ E. B. Stukalin and A. B. Kolomeisky, "Simple growth models of rigid multifilament biopolymers," J. Chem. Phys. 121, 1097-1104 (2004)

${ }^{21}$ T. Ambjörnsson, S. K. Banik, M. A. Lomholt, and R. Metzler, "Master equation approach to DNA breathing in heteropolymer DNA," Phys. Rev. E 75, 021908 (2007)

${ }^{22}$ G. Margolin, H. V. Goodson, and M. S. Alber, "Mean-field study of the role of lateral cracks in microtubule dynamics," Phys. Rev. E 83, 041905 (2011)

${ }^{23}$ C. Li, J. Li, H. V. Goodson, and M. S. Alber, "Microtubule dynamic instability: the role of cracks between protofilaments," Soft Matter 10, 2069-2080 (2014)

${ }^{24}$ M. Muthukumar and A. Baumgaertner, "Effects of entropic barriers on polymer dynamics," Macromolecules 22, 1937-1941 (1989)

${ }^{25} \mathrm{~K}$. S. Schweizer and E. J. Saltzman, "Entropic barriers, activated hopping, and the glass transition in colloidal suspensions," J. Chem. Phys. 119, 1181-1196 (2003).

${ }^{26} \mathrm{~T}$. W. Burkhardt, "Semiflexible polymer in the half plane and statistics of the integral of a Brownian curve," J. Phys. A: Math. Gen. 26, L1157 (1993)

${ }^{2} \mathrm{~T}$. W. Burkhardt, "Free energy of a semiflexible polymer in a tube and statistics of a randomly-accelerated particle," J. Phys. A: Math. Gen. 30, L167 (1997)

${ }^{28}$ A. N. Semenov, "Adsorption of a semiflexible wormlike chain," Eur. Phys. J. E 9, 353-363 (2002)

${ }^{29}$ P. Hänggi, P. Talkner, and M. Borkovec, "Reaction-rate theory: fifty years after kramers," Rev. Mod. Phys. 62, 251-341 (1990)

${ }^{30}$ H. Risken, The Fokker-Planck equation: methods of solution and applications (Springer, Berlin, Heidelberg, 1996).

${ }^{31}$ A. Szabo, K. Schulten, and Z. Schulten, "First passage time approach to diffusion controlled reactions," J. Chem. Phys. 72, 4350-4357 (1980)

${ }^{32}$ R. W. Pastor, R. Zwanzig, and A. Szabo, "Diffusion limited first contact of the ends of a polymer: comparison of theory with simulation," J. Chem. Phys. 105, 3878-3882 (1996).

${ }^{33}$ O. Kratky and G. Porod, "Röntgenuntersuchung gelöster fadenmoleküle," Recl. Trav. Chim. Pays-Bas 68, 1106-1122 (1949)

${ }^{34} \mathrm{M}$. Doi and S. F. Edwards, The theory of polymer dynamics (Clarendon Press, Oxford, 1986).

${ }^{35}$ M. W. Matsen, J. U. Kim, and A. E. Likhtman, "Finite-N effects for ideal polymer chains near a flat impenetrable wall," Eur. Phys. J. E 29, 107-115 (2009)

${ }^{36}$ P. G. de Gennes, "Conformations of polymers attached to an interface," Macromolecules 13, 1069-1075 (1980)

${ }^{37}$ J. F. Joanny, L. Leibler, and P. G. De Gennes, "Effects of polymer solutions on colloid stability," J. Polym. Sci. B 17, 1073-1084 (1979)

${ }^{38}$ T. Bickel, C. Jeppesen, and C. M. Marques, "Local entropic effects of polymers grafted to soft interfaces," Eur. Phys. J. E 4, 33-43 (2001)

${ }^{3 y}$ E. A. DiMarzio, "Proper accounting of conformations of a polymer near a surface," J. Chem. Phys. 42, 2101-2106 (1965)

${ }^{40}$ S. F. Edwards and K. F. Freed, "The entropy of a confined polymer. i," J. Phys. A: Gen. Phys. 2, 145-150 (1969)

${ }^{41}$ H. S. Carslaw and J. C. Jaeger, Conduction of heat in solids (Oxford University Press, Oxford, 1959).

${ }^{42}$ S.-L. Zhao, J. Wu, D. Gao, and J. Wu, "Gaussian fluctuations in tethered DNA chains," J. Chem. Phys. 134, 065103 (2011)

${ }^{43} \mathrm{~S}$. Bell and E. M. Terentjev, "Kinetics of tethered ligands binding to a surface receptor," Macromolecules 50, 8810-8815 (2017) 
${ }^{44}$ V. VanBuren, D. J. Odde, and L. Cassimeris, "Estimates of lateral and longitudinal bond energies within the microtubule lattice," Proc. Natl. Acad. Sci. U.S.A. 99, 6035-6040 (2002)

${ }^{45}$ P. Zakharov, N. Gudimchuk, V. Voevodin, A. Tikhonravov, F. I. Ataullakhanov, and E. L. Grishchuk, "Molecular and mechanical causes of microtubule catastrophe and aging," Biophys. J. 109, 2574-2591 (2015)

${ }^{40}$ D. S. Grebenkov, "First exit times of harmonically trapped particles: a didactic review," J. Phys. A: Math. Theor. 48, 013001 (2015).

${ }^{47} \mathrm{H}$. Brenner, "The slow motion of a sphere through a viscous fluid towards a plane surface," Chem. Eng. Sci. 16, 242-251 (1961)

${ }^{48}$ M. I. M. Feitosa and O. N. Mesquita, "Wall-drag effect on diffusion of colloidal particles near surfaces: A photon correlation study," Phys. Rev. A 44, 6677 (1991)

${ }^{49}$ M. A. Bevan and D. C. Prieve, "Hindered diffusion of colloidal particles very near to a wall: Revisited," J. Chem. Phys. 113,
$1228-1236(2000)$

${ }^{50}$ K. D. Kihm, A. Banerjee, C. K. Choi, and T. Takagi, "Near-wall hindered Brownian diffusion of nanoparticles examined by threedimensional ratiometric total internal reflection fluorescence microscopy," Exp. Fluids 37, 811-824 (2004)

${ }^{51}$ J. Zakrisson, K. Wiklund, O. Axner, and M. Andersson, "The shaft of the type 1 fimbriae regulates an external force to match the fimh catch bond," Biophys. J. 104, 2137-2148 (2013)

${ }^{52} \mathrm{~F}$. Brochard-Wyart, "Deformations of one tethered chain in strong flows," Europhys. Lett. 23, 105-111 (1993)

${ }^{53}$ A. Ferrantini and E. Carlon, "Anomalous zipping dynamics and forced polymer translocation," J. Stat. Mech. Theory Exp. 2011, P02020 (2011)

\section{Appendix A: Derivation of $K_{\mathrm{sf}}^{0}$}

In this section, we calculate the MFPT of the rod-free case to obtain the expression in Eq. (14). The inverse of this MFPT then is the detaching rate which we use in the main context. We will use the non-dimensional variable in our derivations for the benefit of neatness, and then transform the final result back to the dimensional form. We choose the segment length $b$ and the thermal energy $k_{B} T$ as conversion units, writing $\tilde{a}=a / b, \tilde{c}=c / b, \tilde{L}_{p}=L_{p} / b$ and $\tilde{V}_{\mathrm{eff}}^{0}=V_{\mathrm{eff}}^{0} / k_{B} T$. In this way, the non-dimensional form of MFPT integral in Eq. (5) becomes:

$$
\tilde{\tau}_{\mathrm{sf}}^{0} \equiv \frac{\tau_{\mathrm{sf}}^{0} D}{b^{2}}=\int_{\tilde{a}}^{\tilde{c}} e^{\tilde{V}_{\mathrm{sf}}^{0}(\tilde{z})}\left(\int_{0}^{\tilde{z}} e^{-\tilde{V}_{\mathrm{sf}}^{0}(\tilde{x})} d \tilde{x}\right) d \tilde{z}, \quad \text { with } \quad \tilde{V}_{\mathrm{sf}}^{0}(\tilde{z})=\frac{\tilde{k}}{2}(\tilde{z}-\tilde{a})^{2}-\frac{\tilde{L}_{p}}{2}\left[\frac{1}{\sqrt{1+(\tilde{z}-\tilde{a})^{2}}}-1\right]
$$

Under the large- $k$ assumption, we apply the Gaussian approximation to $\operatorname{Exp}\left(-\tilde{V}_{\mathrm{sf}}^{0}\right)$ in the first integral of Eq. A.1), and further replace the integral limits with $\pm \infty$, giving:

$$
\int_{-\infty}^{\infty} \operatorname{Exp}\left[-\frac{1}{4}\left(2 \tilde{k}+\tilde{L}_{p}\right)(\tilde{x}-\tilde{a})^{2}\right] d \tilde{x}=\frac{2 \sqrt{\pi}}{\sqrt{2 \tilde{k}+\tilde{L}_{p}}}
$$

The second integral of Eq. A.1 can be dealt with by Taylor-expanding the exponent of the inside integrand around $\tilde{z}=\tilde{c}$ to the first order (giving an exponential function), because the effective potential increases when the monomer moves towards $\tilde{c}$ from the position $\tilde{a}$, meaning the major contribution is around $\tilde{z}=\tilde{c}$. We further replace the lower integral limit $\tilde{a}$ with $-\infty$, for this approximated exponential function decays fast when $\tilde{z}$ decreases from $\tilde{c}$, altogether giving:

$$
\begin{aligned}
& \int_{-\infty}^{\tilde{c}} \operatorname{Exp}\left\{\frac{\tilde{k}}{2}(\tilde{c}-\tilde{a})^{2}+\frac{\tilde{L}_{p}}{2}\left(1-\frac{1}{\sqrt{1+(\tilde{c}-\tilde{a})^{2}}}\right)+(\tilde{c}-\tilde{a})\left[\tilde{k}+\frac{\tilde{L}_{p}}{2\left(1+(\tilde{c}-\tilde{a})^{2}\right)^{3 / 2}}\right](\tilde{z}-\tilde{c})\right\} d \tilde{z} \\
& =\frac{1}{(\tilde{c}-\tilde{a})\left[\tilde{k}+\frac{\tilde{L}_{p}}{2\left(1+(\tilde{c}-\tilde{a})^{2}\right)^{3 / 2}}\right]} \operatorname{Exp}\left[\frac{\tilde{k}}{2}(\tilde{c}-\tilde{a})^{2}+\frac{\tilde{L}_{p}}{2}\left(1-\frac{1}{\sqrt{1+(\tilde{c}-\tilde{a})^{2}}}\right)\right] \text {. }
\end{aligned}
$$

The product of Eqs. A.2 and A.3 gives the MFPT without the rod:

$$
\tilde{\tau}_{\mathrm{sf}}^{0} \approx \frac{2 \sqrt{\pi} \operatorname{Exp}\left[\frac{\tilde{k}}{2}(\tilde{c}-\tilde{a})^{2}+\frac{\tilde{L}_{p}}{2}\left(1-\frac{1}{\sqrt{1+(\tilde{c}-\tilde{a})^{2}}}\right)\right]}{(\tilde{c}-\tilde{a})\left[\tilde{k}+\frac{\tilde{L}_{p}}{2\left(1+(\tilde{c}-\tilde{a})^{2}\right)^{3 / 2}}\right] \sqrt{2 \tilde{k}+\tilde{L}_{p}}} .
$$

Transforming this $\tilde{\tau}_{\mathrm{sf}}^{0}$ expression back into the dimensional form gives the final result of $\tau_{\mathrm{sf}}^{0}$ in Eq. (14). Without doubt, if one applies Gaussian approximation to the second integral of MFPT calculation (instead of stopping at the 
first order correction as above), a more accurate $\tau_{\mathrm{sf}}^{0}$ expression can be obtained. Here, we only present the final result with this improvement:

$$
\tilde{\tau}_{\mathrm{sf}}^{0} \approx \frac{2 \sqrt{\pi} \operatorname{Exp}\left[\frac{\tilde{k}}{2}(\tilde{c}-\tilde{a})^{2}+\frac{\tilde{L}_{p}}{2}\left(1-\frac{1}{\sqrt{1+(\tilde{c}-\tilde{a})^{2}}}\right)\right]}{(\tilde{c}-\tilde{a})\left[\tilde{k}+\frac{\tilde{L}_{p}}{2\left(1+(\tilde{c}-\tilde{a})^{2}\right)^{3 / 2}}\right] \sqrt{2 \tilde{k}+\tilde{L}_{p}}}\left[1+\frac{\tilde{k}-\frac{\left(2(\tilde{c}-\tilde{a})^{2}-1\right) \tilde{L}_{p}}{2\left(1+(\tilde{c}-\tilde{a})^{2}\right)^{5 / 2}}}{(\tilde{c}-\tilde{a})^{2}\left(\tilde{k}+\frac{\tilde{L}_{p}}{2\left(1+(\tilde{c}-\tilde{a})^{2}\right)^{3 / 2}}\right)^{2}}\right] .
$$

\section{Appendix B: Derivation of $K_{\mathrm{sf}}^{\infty}$}

A similar procedure to $\mathrm{A}$ is applied here, also in the non-dimensional formalism. The effective potential of a long rod is obtained by inserting $\theta_{\min }=0$ into $V_{\text {sf }}$ of Eq. 13 . Its non-dimensional expression takes the form:

$$
\tilde{V}_{\mathrm{sf}}^{\infty}(\tilde{z})=\tilde{V}_{\mathrm{sf}}^{0}(\tilde{z})-\ln \left[1+\operatorname{erf}\left(\frac{\phi}{2} \sqrt{\tilde{L}_{p}}\right)\right]
$$

where all the non-dimensional parameters and variables are defined above Eq. A.1, $\tilde{V}_{\mathrm{sf}}^{0}$ is given in Eq. A.1, and $\phi=\sin ^{-1}\left[(\tilde{z}-\tilde{a}) / \sqrt{1+(\tilde{z}-\tilde{a})^{2}}\right]$, with its expression in terms of dimensional parameters given below Eq. (13).

We put the new effective potential $\tilde{V}_{\mathrm{sf}}^{\infty}$ into the MFPT integral of Eq. A.1. In order to apply the Gaussian approximation to the first integral, we have to first find its saddle point by solving the equation: $\partial \tilde{V}_{\mathrm{sf}}^{\infty} / \partial \tilde{z}=0$. Since $\tilde{k}$ is assumed to be strong, it is expected that this saddle point, $\tilde{z}^{*}$, has only a small shift to the spring potential well $\tilde{z}=\tilde{a}$. We write $\tilde{z}^{*}=\tilde{a}+m$ and put this form into the saddle point equation $\partial \tilde{V}_{\mathrm{sf}}^{\infty} / \partial \tilde{z}=0$. Therein, we further use Taylor-expansion around $m=0$ to first order (since $m$ is only a small shift), and solve the equation of $m$ to obtain the saddle point as:

$$
\tilde{z}^{*}=\tilde{a}+\frac{2 \sqrt{\pi \tilde{L}_{p}}}{2 \tilde{k} \pi+(2+\pi) \tilde{L}_{p}},
$$

which gradually decreases to $\tilde{a}$, when either $\tilde{k}$ or $\tilde{L}_{p}$ increases, meaning that in the case of a strong spring spring or stiff filament, the monomer tends to stay in its equilibrium position of the original potential (without the entropic effect from the rod) as expected.

The Gaussian approximation for the integrand $\operatorname{Exp}\left(-\tilde{V}_{\mathrm{sf}}^{\infty}\right)$, with $\tilde{z}^{*}$ of Eq. B.2 inserted, give the zeroth-oder term as:

$$
\text { 0th order }=\frac{-2 \pi \tilde{k}}{\tilde{L}_{p}\left(2+\pi+\frac{2 \pi \tilde{k}}{\tilde{L}_{p}}\right)^{2}}+\frac{\tilde{L}_{p}}{2}\left(\frac{1}{\sqrt{1+\frac{4 \pi \tilde{L}_{p}}{\left(2 \tilde{L}_{p}+2 \pi \tilde{k}+\pi \tilde{L}_{p}\right)^{2}}}}-1\right)+\ln \left[1+\operatorname{erf}\left(\frac{\sqrt{\pi}}{\sqrt{\left(2+\pi+\frac{2 \pi \tilde{k}}{\tilde{L}_{p}}\right)^{2}+\frac{4 \pi}{\tilde{L}_{p}}}}\right)\right] .
$$

We notice that $4 \pi \tilde{L}_{p} /\left(2 \tilde{L}_{p}+2 \pi \tilde{k}+\pi \tilde{L}_{p}\right)^{2}$ in the second term on the right side of the equation is actually a small term in the large- $\tilde{k}$ regime. Hence the second term can be approached by: $-\pi /\left[2+\pi+2 \pi\left(\tilde{k} / \tilde{L}_{p}\right)\right]^{2}$. Also, inside the erf-function above, $4 \pi / \tilde{L}_{p}$ is negligible compared with the square term in the denominator. Altogether, we simplify the zeroth-order term as:

$$
\text { 0th order } \approx \frac{-\pi\left(2 \tilde{k}+\tilde{L}_{p}\right)}{\tilde{L}_{p}\left(2+\pi+\frac{2 \pi \tilde{k}}{\tilde{L}_{p}}\right)^{2}}+\ln \left[1+\operatorname{erf}\left(\frac{\sqrt{\pi}}{2+\pi+\frac{2 \pi \tilde{k}}{\tilde{L}_{p}}}\right)\right]
$$

The same procedures can be applied to simply the Gaussian term. The result gives a general form Exp[-0.5 $\tilde{k}-$ $\left.A \tilde{L}_{p}(\cdots)+B\right]$, where the last term $B$ cannot extract any $\tilde{k}$ or $\tilde{L}_{p}$ factors out, and is negligible (verified by plotting these three terms against $\tilde{L}_{p}$ at varied $\tilde{k}$ values over a wide range). We will hence drop off this irrelevant term $B$. On the other hand, although the coefficient $A$ has a complicated form, with the ratio $\tilde{k} / \tilde{L}_{p}$ being the only parameter embedded inside, it is a rather flat function which is insensitive to the change of this ratio, and can be described 
very well by the value $3 / 8$. Since all these calculations are straightforward, we only present the final approximated expression here:

$$
\text { Gaussian term } \approx \operatorname{Exp}\left[-\left(\frac{\tilde{k}}{2}+\frac{3 \tilde{L}_{p}}{8}\right)\left(\tilde{z}-\tilde{z}^{*}\right)^{2}\right] .
$$

Combining Eqs. (B.4) and B.5, the first integral in the MFPT becomes:

$$
\int_{-\infty}^{\infty} e^{-\tilde{V}_{\text {eff }}^{\infty}(\tilde{x})} d \tilde{x}=\frac{2 \sqrt{2 \pi}}{\sqrt{4 \tilde{k}+3 \tilde{L}_{p}}}\left[1+\operatorname{erf}\left(\frac{\sqrt{\pi}}{2+\pi+\frac{2 \pi \tilde{k}}{\tilde{L}_{p}}}\right)\right] \operatorname{Exp}\left[\frac{-\pi\left(2 \tilde{k}+\tilde{L}_{p}\right)}{\tilde{L}_{p}\left(2+\pi+\frac{2 \pi \tilde{k}}{\tilde{L}_{p}}\right)^{2}}\right] .
$$

In the second integral of MFPT expression, we Taylor-expand the exponent of the integrand $\operatorname{Exp}\left(\tilde{V}_{\text {eff }}^{\infty}\right)$ around $\tilde{z}=\tilde{c}$ to the first order, giving an exponential function that decays fast when $\tilde{z}$ decreases from $\tilde{c}$. Following this approach, the zeroth-order term for this exponential is:

$$
\begin{aligned}
\text { 0th order }= & \operatorname{Exp}\left\{\frac{\tilde{k}}{2}(\tilde{c}-\tilde{a})^{2}+\frac{\tilde{L}_{p}}{2}\left(1-\frac{1}{\sqrt{1+(\tilde{c}-\tilde{a})^{2}}}\right)-\ln \left(1+\operatorname{erf}\left[\frac{\sqrt{\tilde{L}_{p}}}{2} \sin ^{-1}\left(\frac{\tilde{c}-\tilde{a}}{\sqrt{1+(\tilde{c}-\tilde{a})^{2}}}\right)\right]\right\}\right. \\
& \approx \frac{1}{2} \operatorname{Exp}\left[\frac{\tilde{k}}{2}(\tilde{c}-\tilde{a})^{2}+\frac{\tilde{L}_{p}}{2}\left(1-\frac{1}{\sqrt{1+(\tilde{c}-\tilde{a})^{2}}}\right)\right]
\end{aligned}
$$

where the $\ln$-term is roughly $\ln 2$ with $\tilde{L}_{p}$ larger than 5 , when inserting the practical values for $\tilde{a}$ and $\tilde{c}$ (the parameter range is stated in Sec. II A. On the other side, the first-order expansion of the exponent gives:

$$
\begin{aligned}
& \text { first order }=\operatorname{Exp}\left[(\tilde{c}-\tilde{a})\left[\tilde{k}+\frac{\tilde{L}_{p}}{2\left(1+(\tilde{c}-\tilde{a})^{2}\right)^{3 / 2}}\right]-F\right](\tilde{z}-\tilde{c}), \\
& \text { with } \quad F=\frac{\sqrt{\tilde{L}_{p}} \operatorname{Exp}\left(-\tilde{L}_{p} \phi_{c}^{2} / 4\right)}{\sqrt{\pi}\left[1+(\tilde{c}-\tilde{a})^{2}\right]\left[1+\operatorname{erf}\left(\frac{\sqrt{\tilde{L}_{p}}}{2} \phi_{c}\right)\right]} .
\end{aligned}
$$

$\phi_{c}$ is the $\phi$-angle at $\tilde{z}=\tilde{c}$. The $F$-term is negligible compared with other terms in the exponent, due to its exponentialdecay nature as $\tilde{L}_{p}$ increases. Using this simplification for Eq. (B.8) and together with Eq. (B.7), the second integral for the MFPT expression takes the form:

$$
\int_{-\infty}^{c} e^{\tilde{V}_{\mathrm{sf}}^{\infty}(\tilde{z})} d \tilde{z}=\frac{\operatorname{Exp}\left[\frac{\tilde{k}}{2}(\tilde{c}-\tilde{a})^{2}+\frac{\tilde{L}_{p}}{2}\left(1-\frac{1}{\sqrt{1+(\tilde{c}-\tilde{a})^{2}}}\right)\right]}{2(\tilde{c}-\tilde{a})\left[\tilde{k}+\frac{\tilde{L}_{p}}{2\left(1+(\tilde{c}-\tilde{a})^{2}\right)^{3 / 2}}\right]}
$$

Note that here we have already replaced the original lower integral boundary $\tilde{a}$ with $-\infty$, due to large- $\tilde{k}$ assumption. Combining Eqs. (B.6) and (B.9), we arrive at the expression for the MFPT in a long-rod case:

$$
\tilde{\tau}_{\mathrm{sf}}^{\infty} \approx \sqrt{\frac{2 \tilde{k}+\tilde{L}_{p}}{8 \tilde{k}+6 \tilde{L}_{p}}}\left[1+\operatorname{erf}\left(\frac{\sqrt{\pi}}{2+\pi+\frac{2 \pi \tilde{k}}{\tilde{L}_{p}}}\right)\right] \operatorname{Exp}\left[\frac{-\pi\left(2 \tilde{k}+\tilde{L}_{p}\right)}{\tilde{L}_{p}\left(2+\pi+\frac{2 \pi \tilde{k}}{\tilde{L}_{p}}\right)^{2}}\right] \tilde{\tau}_{\mathrm{sf}}^{0} .
$$

We examine this pre-factor over a wide range of $\tilde{k}$ and $\tilde{L}_{p}$ values, while setting $1 \leq \tilde{a} \leq 1.5$ and $2 \leq \tilde{c} \leq 3$, and discover that this factor is almost a fixed constant sitting between 0.497 and 0.505 , insensitive to the variation of these parameters. We therefore substitute the value 0.5 for this pre-factor, obtaining the expression used in the main context: $\tau_{\mathrm{sf}}^{\infty} \approx \tau_{\mathrm{sf}}^{0} / 2$ and hence $K_{\mathrm{sf}}^{\infty} \approx 2 K_{\mathrm{sf}}^{0}$.

\section{Appendix C: Numerical comparison of $K_{\mathrm{sf}}$}

In this section, we compare the numerical calculation and approximation for acceleration pre-factor $\left(K_{\mathrm{sf}} / K_{\mathrm{sf}}^{0}\right)$ from Eq. 15. We specifically focus on the $L_{p}$-effect on the error, while choosing $b=1, a=1$ and $c=2$ nm and 

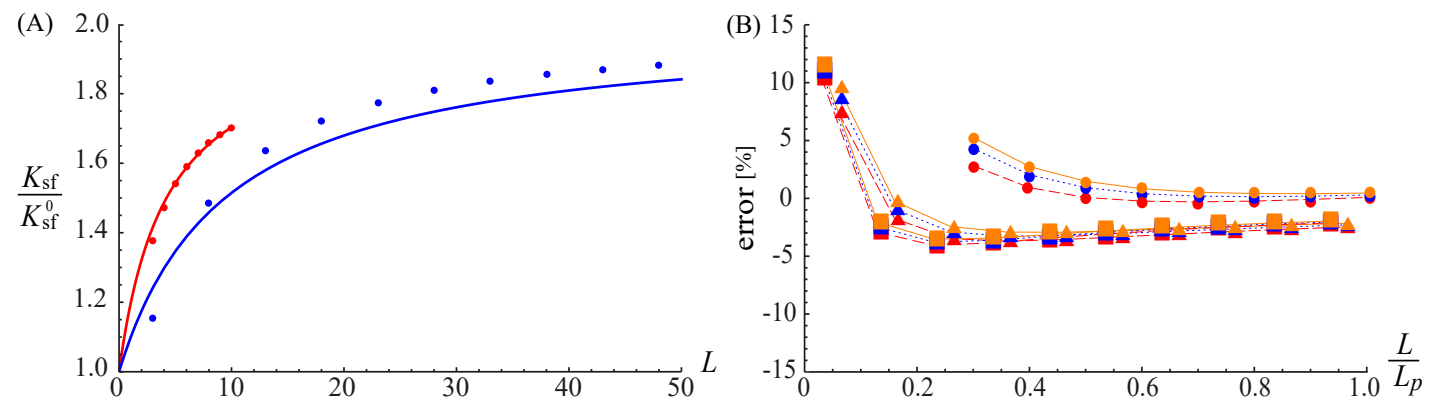

FIG. 7. (A) The acceleration ratio, $K_{\mathrm{sf}} / K_{\mathrm{sf}}^{0}$, plotted against the rod length $L$, with $L_{p}=50$ nm (rigid filament, upper curve) and $10 \mathrm{~nm}$ (less stiff filament, lower curve). Numerical results are shown as dots, while solid lines are produced by Eq. (15). (B) The percentage error for $K_{\mathrm{sf}} / K_{\mathrm{sf}}^{0}$, plotted against the scaled rod length $L / L_{p}$, at different spring strength $k$ and persistence length $L_{p}$. The squares, triangles, and circles are estimated at $L_{p}=100,50$ and $10 \mathrm{~nm}$, while with $k=10$ (connected by the red dashed line), 15 (blue dotted), 20 (orange solid) $\mathrm{k}_{\mathrm{B}} \mathrm{T} \cdot \mathrm{nm}^{-2}$.

keeping these values fixed. In order to examine solely the accuracy of the approximation for the $\left(K_{\mathrm{sf}} / K_{\mathrm{sf}}^{0}\right)$-ratio, we deliberately compute both $K_{\mathrm{sf}}$ and $K_{\mathrm{sf}}^{0}$ numerically (by inserting the effective potential of Eqs. (11) and (13) into the MFPT integral, respectively), instead of using the approximation for $K_{\mathrm{sf}}^{0}$, namely, Eqs. (14) or (A.5) which may be another source of error. We plot the numerical results and the approximation Eq. (15) against rod length $L$ on Fig. 7(A), at fixed spring strength $k=10 \mathrm{k}_{\mathrm{B}} \mathrm{T} \cdot \mathrm{nm}^{-2}$ such that the bond energy is $5 \mathrm{k}_{\mathrm{B}} \mathrm{T}$, for a rigid and a less stiff filament $\left(L_{p}=50\right.$ and $\left.10 \mathrm{~nm}\right)$. We remind that the effective potential in the rod-like model only holds in the regime $c<L$, see the argument in Sec. IIIA. We do not have the needed potential in the MFPT integral, to estimate the unbinding rate $K_{\mathrm{sf}}$ in the regime $0<L<c$. Therefore, the numerical results in this region $L \leq c$ will not be showed. Figure 7(A) shows that our approximation does capture the trend of the numerical curves, and that the deviation is the largest at $L \approx c$ and decreases as $L$ increases. The cause for this phenomenon (higher error occurring near $L=c$ ) is due to the limitation of using interpolation method to cover the region $0<L<c$.

To further explore the magnitude of the error, we divide the ratio $\left(K_{\mathrm{sf}} / K_{\mathrm{sf}}^{0}\right)$ given in Eq. (15) by the numerical results estimated from direct MFPT integral, and plot its error percentage against the scaled rod length, $L / L_{p}$, in three groups of different $k$ and $L_{p}$ values, on Fig. 7(B). Within each group, the $L_{p}$ value is held the same and only $k$-values are changed to produce three curves. We choose $L_{p}=10,50$ and $100 \mathrm{~nm}$, with $k=10,15$ and $20 \mathrm{k} \mathrm{B} \cdot \mathrm{nm}^{-2}$, to cover the range of the bond energy commonly seen in biological systems (from 5 to $10 k_{B} T$ ). Figure 7(B) shows that the error of our approximation is almost within $5 \%$, except for the point near $L=c$ (10\% error there), for all curves. The increase of filament rigidity seems to increase error percentage, and one may worry that this approximation is not suitable beyond the range of $L_{p}$-values we investigate. However, it shall not be a major concern, because the persistence length $L_{p}$ of $100 \mathrm{~nm}$ will contribute to a bending penalty of $27.64 \mathrm{k}_{\mathrm{B}} \mathrm{T}$ to unbind (estimated by using the bending energy term inside Eq. (11) with $z=c$ ) without the assistance of external pulling forces. This energy penalty is so high that one would expect this reaction unlikely to occur, or simply too slow to be an important kinetic process. And even at such large- $L_{p}$ value $\left(L_{p}=100 \mathrm{~nm}\right)$, our approximation is still within $10 \%$ error. 